\title{
On $C^{r}$-closing for flows on 2-manifolds.
}

\author{
Carlos Gutierrez \\ e-mail: gutp@impa.br \\ IMPA. Estrada Dona Castorina 110, J. Botânico, \\ 22460-320, Rio de Janeiro, R.J., Brazil.
}

\begin{abstract}
For some full measure subset $\mathcal{B}$ of the set of iet's (i.e. interval exchange transformations) the following is satisfied:

Let $X$ be a $C^{r}, 1 \leq r \leq \infty$, vector field, with finitely many singularities, on a compact orientable surface $M$. Given a nontrivial recurrent point $p \in M$ of $X$, the holonomy map around $p$ is semi-conjugate to an iet $E:[0,1) \rightarrow[0,1)$. If $E \in \mathcal{B}$ then there exists a $C^{r}$ vector field $Y$, arbitrarily close to $X$, in the $C^{r}$-topology, such that $Y$ has a closed trajectory passing through $p$.
\end{abstract}

\section{Introduction}

The open problem " $C^{r}$-closing lemma" is stated as follows:

"Let $M$ be a smooth compact manifold, $r \geq 2$ be an integer, $f \in \operatorname{Diff}^{r}(M)$ (resp. $\left.X \in \mathfrak{X}^{r}(M)\right)$ and $p$ be a nonwandering point of $f$ (resp. of $X$ ). There exists $g \in \operatorname{Diffr}^{r}(M)$ (resp. $\left.Y \in \mathfrak{X}^{r}(M)\right)$ arbitrarily close to $f$ (resp. to $X$ ) in the $C^{r}$-topology so that $p$ is a periodic point of $g$ (resp. of $Y$ )".

C. Pugh proved the $C^{1}$-closing lemma [Pg1]. There are few previous results when $r \geq 2$ : Gutierrez [Gu1] showed similar results to this paper when the manifold is the torus $T^{2}$. There are negative answers: Gutierrez [Gu3] proved that if the perturbation is localized in a small neighborhood of the nontrivial recurrent point, then $C^{2}$-closing is not always possible. C. Carroll's [Car] proved that, even for flows with finitely many singularities, $C^{2}$-closing 
by a twist-perturbation (supported in a cylinder) is not always possible. Concerning hamiltonian flows, M. Herman [Her] has remarkable counter-examples to the $C^{r}$-closing lemma. Within the context of geodesic laminations, S. Aranson and E. Zhuzhoma anounced in 1988 [A-Z] the $C^{r}$-closing lemma for a class of flows on surfaces; however, their proofs have not been published yet. For basic definition the reader may consult $[\mathrm{K}-\mathrm{H}]$.

\section{Statement of the results}

Throughout this article, $M$ will be a smooth, orientable, compact, two manifold and $\chi$ will be its Euler characteristic. We shall denote by $\mathfrak{X}^{r}(M)$ the space of vector field of class $C^{r}, 1 \leq r \leq \infty$, with the $C^{r}$-topology. The trajectory of $X \in \mathfrak{X}^{r}(M)$ passing through $p \in M$ will be denoted by $\gamma_{p}$ The domain of definition of a map $S$ will be denoted by $\operatorname{DOM}(S)$. Smooth segments on $M$ will be denoted and referred as (open, half-open, closed) intervals.

A bijective map $E:[0,1) \rightarrow[0,1)$ is said to be an iet, i.e. an Interval Exchange Transformation (with $m$ intervals) if there exists a finite sequence $0=a_{1}<a_{2}<\cdots<a_{m}<a_{m+1}=1$ such that, for all $i \in\{1,2, \cdots, m\}$ and for all $x \in\left[a_{i}, a_{i+1}\right), E(x)=E\left(a_{i}\right)+x-a_{i}$, and moreover, $E$ is discontinuous at exactly $a_{2}, a_{3}, \cdots, a_{m}$. This $E$ will be identified with the pair $(\lambda, \pi) \in \Delta_{m} \times$ $\mathfrak{S}_{m}$ made up of the positive probability vector $\lambda=\left\{\left|a_{i+1}-a_{i}\right|\right\}_{i=1}^{m}$ and the permutation $\pi$ on the symbols $1,2, \cdots, m$, defined by $\pi(i)=\#\left\{j: E\left(a_{j}\right) \leq\right.$ $\left.E\left(a_{i}\right)\right\}$. The space of iet's, with $m$ intervals, defined in $[0,1)$, will be identified with the measurable space $\Delta_{m} \times \mathfrak{S}_{m}$ endowed with the product measure, where $\Delta_{m}$ is the simplex of positive probability vectors of $\mathbb{R}^{m}$, with Lebesgue measure, and $\mathfrak{S}_{m}$ is the finite set of permutations on $m$ symbols with counting measure. Let $E:[a, b) \rightarrow[a, b)$ be an iet. We say that $[s, t] \subset[a, b)$ is a virtual orthogonal edge for $E$, if $E$ restricted to $[s, t]$ is continuous and $s<E(s)<E^{2}(s)=t$. Given $k \in \mathbb{N}$, let $\mathcal{B}_{k}$ be the set of iet's $E:[a, b) \rightarrow[a, b)$ such that for some sequence $b_{n} \rightarrow a$ of points of $(a, b)$, and for every $n \in \mathbb{N}$, the iet $E_{n}:\left[a, b_{n}\right) \rightarrow\left[a, b_{n}\right)$, induced by $E$, has at least $\chi+k+3$, pairwise disjoint, virtual orthogonal edges. Denote $\mathcal{B}=\cap_{k \geq 1} \mathcal{B}_{k}$. It will be seen that, as a direct consequence of the work of W. A. Veech [Vee] and H. Masur [Mas],

Theorem 2.1. For all $m \geq 2, \quad \Delta_{m} \times \mathfrak{S}_{m} \backslash \mathcal{B}$ is a measure zero set.

By transporting information along flow boxes, Item (a2) below follows from the definition of $\mathcal{B}_{K}$. 
Theorem 2.2. ([Gu2, Structure Theorem, Section 3]) Let $X \in \mathfrak{X}^{1}(M)$. There are finitely many nontrivial recurrent trajectories $\gamma_{p_{1}}, \gamma_{p_{2}}, \cdots, \gamma_{p_{\ell}}$ of $X$ such that if $\gamma_{p}$ is any nontrivial recurrent trajectory of $X$, then $\overline{\gamma_{p}}=\overline{\gamma_{p_{i}}}$, for some $i=1,2, \cdots, \ell$.

Suppose that $X$ has exactly $K \in \mathbb{N}$ singularities ( $K=0$ is allowed). Let $p \in M$ be a nontrivial recurrent point of $X$. Take a half-open interval $[p, q) \subset M$ transversal to $X$, such that $p$ is a cluster point of $\gamma_{p} \cap(p, q)$, Denote by $P_{X}$ : $[p, q) \rightarrow[p, q)$ the forward Poincaré map induced by $X$. If $[p, q)$ is small enough, it can be associated to $(p,[p, q))$, an iet $E=E_{(p,[p, q))}:[0,1) \rightarrow[0,1)$ and $a$ continuous monotone surjective map $h:[p, q) \rightarrow[0,1)$ such that $h(p)=0$, $h$ restricted to any given orbit of $P_{X}$ is injective and, for all $x \in \operatorname{DOM}\left(P_{X}\right)$, $E \circ h(x)=h \circ P_{X}(x)$; moreover,

(a1) there exists a subset $S \subset[0,1)$ of at most $\chi+K+2$ elements such that if $A$ is a connected component of $[0,1) \backslash S$, then $h^{-1}(A)$ is contained in $\operatorname{DOM}(T)$;

(a2) Let $\bar{p} \in \overline{\gamma_{p}}$ be a nontrivial recurrent point of $X$ and $(\bar{p},[\bar{p}, \bar{q}))$ be a pair satisfying the same conditions as those of $[p,[p, q))$ above. Then the property that the iet $E_{(\bar{p},(\bar{p}, \bar{q}))}$ belongs to $\mathcal{B}_{K}$ does not depend on $(\bar{p},[\bar{p}, \bar{q}))$.

Under conditions of theorem above and if $E \in \mathcal{B}_{K}$, any nontrivial recurrent point of $\overline{\gamma_{p}}$ is said to be of $\mathcal{B}_{K}$-type. Our result is the combination of Theorems 2.1 - 2.3.

Theorem 2.3. Let $X \in \mathfrak{X}^{r}(M), 1 \leq r \leq \infty$, have $K \geq 0$ singularities. Let $p \in M$ be a $\mathcal{B}_{K}$-type nontrivial recurrent point of $X$. Then there exists $Y \in \mathfrak{X}^{r}(M)$, arbitrarily close to $X$, having a closed trajectory passing through $p$.

Related to this theorem (see [Gu2]), we have that: For any $E \in \mathcal{B}$, it can be constructed $Y \in \mathfrak{X}^{\infty}(S)$, for some surface $S$, having a nontrivial recurrent point $p_{0}$ such that item (a1) is satisfied for some $h:\left[p_{0}, q_{0}\right) \rightarrow[0,1)$, and $P_{Y}:\left[p_{0}, q_{0}\right) \rightarrow\left[p_{0}, q_{0}\right)$. Here, $P_{Y}$ can be obtained to be injective or not.

\section{Proof of the results}

Suppose that $M$ is endowed with an orientation and with a smooth riemannian metric $<,>$. Given a $X \in \mathfrak{X}^{r}(M), 1 \leq r \leq \infty$, we define $X^{\perp} \in \mathfrak{X}^{r}(M)$ 


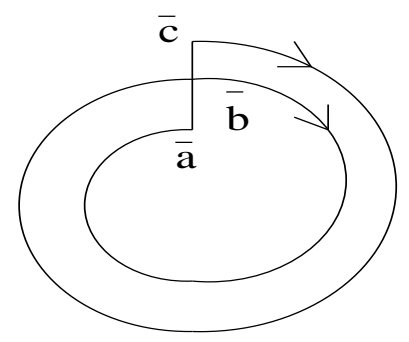

Fig. 1.a

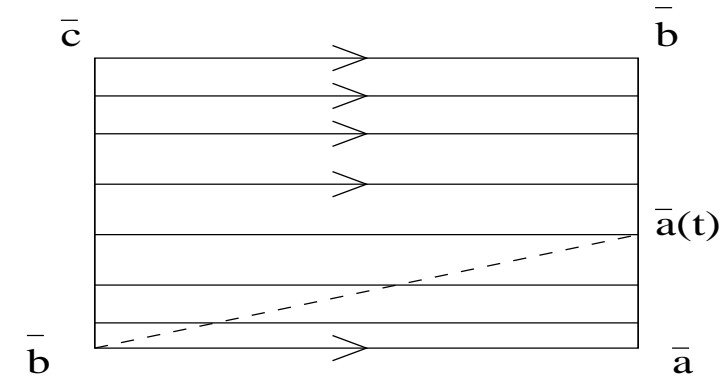

Fig. 1.b

by the following conditions: (a) $\quad<X, X>=<X^{\perp}, X^{\perp}>$; and

when $p \in M$ is regular point of $X$, the ordered pair $\left(X(p), X^{\perp}(p)\right)$ is an orthogonal positive basis of $T_{p}(M)$ (according to the given orientation of $M$ ). let $\Sigma$ be an arc of trajectory of $X^{\perp}$. A $\Sigma$-flow-box (for $X$ ) is a compact subset $F \subset M$ whose interior is a flow box of $X$ and whose boundary $\partial F$ is a graph, homeomorphic to the figure " 8 ", which is the union of arcs of trajectory $[\bar{c}, \bar{a}]_{X}$ and $[\bar{a}, \bar{c}]$ (connecting $\bar{a}$ and $\bar{c}$ ) of $X$ and $X^{\perp}$, respectively. We shall refer to $[\bar{a}, \bar{c}]\left(\right.$ resp. $\left.[\bar{c}, \bar{a}]_{X}\right)$ as the orthogonal (resp. tangent) edge of either $F$ or $\partial F$. See Figs. 1.a and 1.b.

Let $X \in \mathfrak{X}^{r}(M), 1 \leq r \leq \infty$, and let $p \in M$ be a nontrivial recurrent point of $X$. We say that $X$ is T-closable at $p$ (i.e. twist-closable at $p$ ) if there exists a half-open interval $\Sigma=[p, q)$ tangent to $X^{\perp}$, such that, for any neighborhood $V$ of $p$, there exists a $\Sigma$-flow-box for $X$ having its orthogonal edge contained in $\Sigma \cap V$.

Proposition 3.1. Let $X \in \mathfrak{X}^{r}(M), 1 \leq r \leq \infty$, and let $p \in M$ be a nontrivial recurrent point of $X$. Suppose that $X$ is T-closable at $p$. Then there are sequences $t_{n} \rightarrow 0$, of real numbers, and $p_{n} \rightarrow p$, of points of $M$, such that $X+t_{n} X^{\perp}$ has a closed trajectory through $p_{n}$

Proof: As $X$ is T-closable at $p$, there exists a half-open interval $\Sigma=[p, q)$ tangent to $X^{\perp}$, such that, given neighborhoods $\mathcal{V}$ of $X$ and $V$ of $p$, we may choose a $\Sigma$-flow-box $F \subset M$ (for $X)$ and $\sigma>0$ such that if $[\bar{c}, \bar{a}]_{X}$ and $[\bar{a}, \bar{c}]$ ) are the tangent and orthogonal edges, respectively, of $\partial F$, and $\bar{b}$ is the vertex of $\partial F$, then:

(b1) $[\bar{a}, \bar{c}] \subset V$ and the flow of $X$ enters into $F$ through the closed subinterval $[\bar{b}, \bar{c}]$ of $\Sigma$; moreover, for all $t \in[-\sigma, \sigma], X(t):=X+t X^{\perp} \in \mathcal{V}$; 
(b2) both $X(\sigma)$ and $X(-\sigma)$ have an arc of trajectory contained in $F$, which is a global cross section for $\left.X\right|_{F}$.

We shall continue considering only the case in which the flow of $X^{\perp}$ goes from $\bar{a}$ to $\bar{c}$. Let $\Gamma$ be the set of real numbers $s \in[0, \sigma]$ such that when $t \in[0, s]$ there is an arc of trajectory $[\bar{b}, \bar{a}(t)]_{X(t)}$ of $X(t)$, joining $\bar{b}$ with $\bar{a}(t) \in[\bar{a}, \bar{b}]$, contained in $F$, with $\bar{a}(0)=\bar{a}$, and such that $\bar{a}(t)$ depends continuously on $t$. When $t \in \Gamma$, these conditions determine $\bar{a}(t)$ and also that $[\bar{b}, \bar{a}(t)]_{X(t)}$ is transversal to $X$. Therefore, by (b2), $\Gamma=\left[0, \sigma_{1}\right]$ is a closed interval, $\bar{a}\left(\sigma_{1}\right)=b$ and $\left[\bar{b}, \bar{a}\left(\sigma_{1}\right)\right]_{X\left(\sigma_{1}\right)}$ is a closed trajectory of $X\left(\sigma_{1}\right)$. See Fig. 1.b

Under the assumptions and conclusions of this proposition, there exists a sequence $F_{n}: M \rightarrow M$ of $C^{r}$-diffeomorphisms, taking $p_{n}$ to $p$. We may assume that $F_{n}$ converges to the identity diffeomorphism in the $C^{r+1}$-topology. Therefore, the sequence of vector fields $\left(F_{n}\right)_{*}\left(X+t_{n} X^{\perp}\right) \rightarrow X$ in the $C^{r}$-topology and each $\left(F_{n}\right)_{*}\left(X+t_{n} X^{\perp}\right)$ has a closed trajectory passing through $p$. This proves the following

Theorem 3.2. Let $X \in \mathfrak{X}^{r}(M), 1 \leq r \leq \infty$. Let $p \in M$ be a nontrivial recurrent point of $X$. Suppose that that $X$ is T-closable at $p$. Then there exists $Y \in \mathfrak{X}^{r}(M)$ arbitrarily close to $X$ having a closed trajectory through $p$.

Proof of Theorem 2.1: We shall prove that: For all $m \geq 2, \quad \Delta_{m} \times \mathfrak{S}_{m} \backslash \mathcal{B}$ is a measure zero set. It was proved by W. A. Veech [Vee] and H. Masur [Mas] that the Rauzy operator $\mathcal{R}: \mathcal{M} \rightarrow \mathcal{M}$, defined in a full measure subset $\mathcal{M}$ of $\Delta_{m} \times \mathfrak{S}_{m}$, is ergodic and has the following property:

(c) Given $E \in \mathcal{M}$, there exists a sequence $\left\{\left[0, a_{n}\right)\right\}$ of subintervals of $[0,1)$ such that $a_{n} \rightarrow 0$ and, if $\tilde{E}_{n}:\left[0, a_{n}\right) \rightarrow\left[0, a_{n}\right)$ denotes the iet induced by $E$, then, up to re-scaling, $\mathcal{R}^{n}(E)$ coincides with $\tilde{E}_{n}$; more precisely, $\mathcal{R}^{n}(E)(z)=\left(1 / a_{n}\right) \tilde{E}_{n}\left(a_{n} z\right)$, for all $z \in[0,1)$.

Given $k \geq 1$, let $A_{k}$ be the set of $E \in \Delta_{m} \times \mathfrak{S}_{m}$ such that for some $a \in\left(16^{-k}-32^{-k}, 16^{-k}+32^{-k}\right), E(x)=a+x$, for all $x \in[0,1 / 2]$. We observe that $A_{k}$ is open and so it has positive measure. Let $\tilde{\mathcal{B}}_{k}$ be the set of $E \in \mathcal{M}$ such that the positive $\mathcal{R}$-orbit of $E$ visits $A_{k}$ infinitely many often. As $A_{k}$ has positive measure and $\mathcal{R}$ is ergodic, the complement of $\tilde{\mathcal{B}}_{k}$ has measure zero. Therefore, the complement of $\tilde{\mathcal{B}}=\cap_{k \geq 2} \tilde{\mathcal{B}}_{k}$ has measure zero. Observe that if and iet $E \in A_{k}$, then $E$ has more than $k$, pairwise disjoint, virtual orthogonal edges. Therefore, as $\mathcal{R}$ satisfy (c) right above and since the positive $\mathcal{R}$-orbit 
of any given $E \in \tilde{\mathcal{B}}$ visits every $A_{k}$ infinitely many often, we obtain that $\tilde{\mathcal{B}} \subset \mathcal{B}$. this proves the theorem.

Proof of Theorem 2.3: This theorem is stated as follows: Let $p \in M$ be a $\mathcal{B}_{K}$-type nontrivial recurrent point of $X \in \mathfrak{X}^{r}(M), 1 \leq r \leq \infty$. Suppose that $X$ has $K \geq 0$ singularities. Then there exists a $Y \in \mathfrak{X}^{r}(M)$ arbitrarily close to $X$, having a closed trajectory passing through $p$.

By theorem 3.2, it is enough to prove that $X$ is T-closable at $p$. Let $\Sigma=$ $[p, q), T:[p, q) \rightarrow[p, q), E:[0,1) \rightarrow[0,1), h:[p, q) \rightarrow[0,1)$ be as in Theorem 2.2. As $E \in \mathcal{B}_{K}$, given a neighborhood $V$ of $p$, there exist $b \in(0,1)$ and an iet $E_{V}:[0, b) \rightarrow[0, b)$, such that:

(e) $E_{V}$ has at least $\chi+K+3$ pairwise disjoint virtual orthogonal edges contained in $[0, b)$; moreover, the interval $\Sigma_{V}=h^{-1}([0, b))$ is contained in $V$.

Let $T_{V}: \Sigma_{V} \rightarrow \Sigma_{V}$ be the map induced by $T$. As $X$ has $K$ singularities, (e) and Theorem 2.2 imply that $E_{V}$ has a virtual orthogonal edge $\left[a, E_{V}(a)\right] \subset$ $[0, b)$ such that, for some $\bar{a} \in \operatorname{DOM}\left(\left(T_{\Sigma_{V}}\right)\right), \quad\left[\bar{a}, T_{V}(\bar{a})\right]=h^{-1}([a, E(a)]) \subset$ $\operatorname{DOM}\left(\left.T\right|_{\Sigma_{V}}\right)$. Therefore, there exists a $\Sigma$-flow-box bounded by $\left[\bar{a}, T_{V}^{2}(\bar{a})\right] \cup$ $\left[\bar{a}, T_{V}^{2}(\bar{a})\right]_{X}$. As $V$ is arbitrary, this proves that $X$ is T-closable at $p$.

\section{References}

[A-Z] S. Aranson and E. Zhuzhoma. On the $C^{r}$-closing lemma on surfaces. Russian Math. Surv., 43, 1988, 5, 209-210.

[Car] C. Carroll. Rokhlin towers and $C^{r}$ closing for flows on $T^{2}$. Erg. Th. and Dynam. Sys., 12, 1992, 683-706.

[Gu1] C. Gutierrez. On the $C^{r}$-closing lemma for flows on the torus $T^{2}$. Erg. Th. and Dyn. Sys. (1986), 6, 45-56.

[Gu2] C. Gutierrez. Smoothing continuous flows on two-manifolds and recurrences. Erg. Th. and Dyn. Sys. (1986), 6, 17-44.

[Gu3] C. Gutierrez. A counter-example to a $C^{2}$-closing lemma. Erg. Th. and Dyn. Sys. (1987), 7, 509-530. 
[Her] M. Herman. Exemples de flots hamiltoniens dont aucune perturbation en topologie $C^{\infty}$ n'a d'orbites périodiques sur un ouvert de surfaces d'énergies. C. R. Acad. Sci. Paris, t. 312, Série I (1991) 989-994.

[K-H] A. Katok and B. Hasselblatt Introduction to the Modern Theory of Dynamical Systems. Cambridge University Press, New York (1995).

[Mas] H. Masur. Interval exchange transformations and measured foliations. Ann. Math. 115 (1982), 169-200.

[Pg1] C. Pugh. An improved closing lemma and a general density theorem. Amer. Jour. math., 89 (1967), 1010-1021.

[Vee] W. Veech. Gauss measures for transformations on the space of interval exchange maps. Ann. Math. 115 (1982), 201-242. 